\section{What are the ideal characteris- tics of a venous stent?}

\author{
Ilaria Massi, Paolo Zamboni \\ Center for Veins and Lymphatics \\ Diseases, University Hospital of Ferrara, \\ Cona (FE), Italy
}

\begin{abstract}
Historically, the stents used in the venous system were not dedicated scaffolds. They were largely adapted arterial stents. An essential feature of a venous stent is compliance, in order to adapt its crosssectional area to the vein. It should also be crush resistant, corrosion resistant and fatigue resistant. The material should be radiopaque, for follow-up. Another characteristic of the ideal venous stent is flexibility, to adapt its shape to the vein, not vice versa. The scaffold should be uncovered too, in order to avoid the occlusion of collaterals. The ideal venous stent should not migrate, so it is necessary a large diameter and a long length. The radial force is important to prevent migration. However, current stents derived from arterial use display high radial force, which could affect the patency of the thin venous wall. Alternatively, if the stent has an anchor point, that permits a passive anchoring, the radial force required to avoid migration will be lower. Dedicated venous stents were not available until very recently. Furthermore, there is a preclinical study about a new compliant nitinol stent, denominated Petalo CVS. Out of the commonest causes of large veins obstruction, dedicated venous stent could also treat other diseases described more recently, such as the jugular variant of the Eagle syndrome, JEDI syndrome and jugular lesions of the chronic cerebrospinal venous insufficiency that result unfavorable for angioplasty according to Giaquinta classification.
\end{abstract}

\section{Introduction}

Unlike arteries, veins have higher compliance, while the pressure is lower. Postural changes, respiratory and cardiac cycle, outside and inside pressure modify the cross-sectional area of the veins. Unlike the arteries, the venous vessels have valves to prevent reflux. Chronic obstructive venous disease can be caused by nonthrombotic vein lesions, because of extrinsic compression, such as in the May Thurner syndrome, or endoluminal defects, e.g. stenosis because of congenital defects, or by post-thrombotic fibrosis inside and around the vein. This lesion is different from the atherosclerotic one. So there are differences between arterial and venous diseases (Table 1). Therefore, stents must not be the same. ${ }^{1-3}$

There are more studies about the arterial system than the venous system. The stents currently used in the venous system are not dedicated scaffolds, because they are arterial stents, modified to have more flexibility. They keep, however, a high radial-force and an unchangeable cross-sectional area. . $^{1,4,5}$

The high pressure and the compromised return of blood through several mechanisms are the most important elements that cause obstructive venous disease. ${ }^{6,7}$ Chronic venous insufficiency due to obstruction can be treated with percutaneous venous balloon angioplasty and stenting. ${ }^{8}$ In the past endovenous balloon angioplasty was the most important method to treat occlusive venous disease. It is a safe, minimally invasive technique, but with a low patency rate. So clinical improvement is shorter than expected. . $^{3,-12}$

Currently, occlusive venous disease can be treated with a stent. ${ }^{9-13}$ Venous stent is safe, effective and minimally invasive., $3,711-$ 12,14-31

Early complications, such as hematoma, arterial injury, pseudoaneurysm, nerve injury, are infrequent $(<1 \%){ }^{18}$ The stent's fracture in the venous system is uncommon. ${ }^{16}$ A meta-analysis about the patency rate after ilio-caval stent angioplasty reveals that the primary and secondary patency is $96 \%$ and $99 \%$ for non-thrombotic and $79 \%$ and $94 \%$ for chronic postthrombotic. ${ }^{11,23}$ In the jugular system the rate of thrombotic complication is higher when arterial-derived stents have been implanted. Internal jugular vein thrombosis rarely follows balloon angioplasty, while instent thrombosis occurs in $1.6 \%$ of the cases. ${ }^{19-20,32}$ Internal jugular vein thrombosis is often an underestimated clinical problem, and its more common symptoms are neck pain and headache. ${ }^{32}$ Another long-term complication can be represented by fibrotic tissue that causes extrinsic compression and stent migration. ${ }^{18}$

Currently, the most important stent used in venous surgery is the Wallstent. ${ }^{4}$ Quite recently other venous stents became available on the market, such as Zilver Vena stent, Vici venous stent and Sinus venous stent. ${ }^{27,33-36}$

\section{Venous stent versus arterial stent}

Stents used in the venous system are
Correspondence: Ilaria Massi, Center for Veins and Lymphatics Diseases, University Hospital of Ferrara, via Aldo Moro 8, 44124 Cona (FE), Italy.

E-mail: ilaria.massi@edu.unife.it

Key words: Venous obstruction; venous stent; venous compliance; vein defects.

Contributions: PZ, design and conceptualization; IM, literature research and manuscript writing.

Conflict of interests: the authors declare no potential conflict of interests.

Received for publication: 9 March 2021.

Accepted for publication: 15 June 2021.

This work is licensed under a Creative Commons Attribution 4.0 License (by-nc 4.0).

(C) Copyright: the Author(s), 2021

Licensee PAGEPress, Italy

Veins and Lymphatics 2021; 10:9739

doi:10.4081/vl.2021.9739

largely ideated to treat arterial disease. The knowledge derived from the arterial experience is useful, but endovascular venous techniques cannot be the same because of differences between arteries and veins. The ideal characteristics of a venous stent are known (Table 2). ${ }^{3-4,11,22,37}$

The principal aim of a venous stent is to decrease venous hypertension and re-establish physiological blood flow. ${ }^{1-2,4,11,22,35,38,39}$

Venous scaffold must have a larger diameter and longer length than the same stent used in arteries, to prevent recoil. The oversized diameter increases the radial force, which helps to anchor the stent passively. Currently, the diameter of the stents is 2-4 $\mathrm{mm}$ oversized. Veins' blood pressure is lower than in arteries, so the radial force should be higher to prevent migration. However, current stents derived from arterial use display high radial force, which could affect the patency of the thin venous wall. The high radial force can cause inflammatory response and fibrosis. Alternatively, if the stent has an anchor point, that permits a passive anchoring, the radial force required to avoid migration will be lower and the risk of myointimal hyperplasia and restenosis will decrease too. $1,2,4,11,22,35,38,40,41$

Undersized stent and residual stenoses at the inflow or outflow segments can induce significant complications, such as chronic occlusion or persistence of symptoms. ${ }^{1,3,42}$ The ideal venous stent has not instent restenosis. ${ }^{4}$ In $3.5 \%-10 \%$ of cases the large diameter of the stent promotes a permanent over dilatation of the vein that 
Table 1. The most important differences between vein and artery.

\begin{tabular}{lll} 
& Vein & \\
Compliance & High & Artery \\
Elasticity & High & Low \\
\hline Pressure & Low & High \\
Valves to prevent reflux & Present & Absent \\
\hline Important changes of the cross-sectional area because of postural changes & Present & Absent \\
Lesion & Post-thrombotic fibrosis & Atherosclerotic lesion \\
\hline
\end{tabular}

Table 2. Characteristics of the ideal venous stent.

\begin{tabular}{ll}
\hline Compliant & It can be placed across joints \\
Resistant & No turbulences of blood flow \\
\hline Flexible & No alteration of the wall vessel \\
Radiopaque & No in-stent restenosis \\
\hline Uncovered & A sufficiently high radial force \\
Corrosion-resistant & Large diameter \\
\hline Passive anchor & Long length \\
\hline
\end{tabular}

increases the risk of circumferential thrombosis.

Unlike the arterial system, the vein's diameter is not stable because of postural changes of the body, which induce a continuous modification of hydrostatic pressure, and systemic conditions. Some veins are subjected to pulsations, such as the internal jugular vein. This phenomenon, called jugular venous pulse, is represented by the changes in cross-sectional area. It is generated by atrial pressure during the cardiac pump, and it is transmitted along the vein. The jugular venous pulse is composed of three positive $(a, c, v)$ and two negative waves $(x, y)$. The higher positive wave is $a$, which corresponds to the $p$ wave of the ECG, and it is caused by the atrial contraction. The following waves are $c, x, v$ and $y$, respectively caused by the tricuspid closure $(c)$, its downward movement $(x)$, the ventricular contraction $(v)$ and the opening of the tricuspid valve $(y) \cdot{ }^{1,43-45}$ An essential feature of a venous stent is compliance, to adapt its cross-sectional area to variation of vein's volume and circumference. The stents used currently in the venous system do not have these characteristics, because they are studied as arterial stents. They are not as compliant as necessary. The mechanical properties and the compliance of the venous wall decrease because of the placement of arterial stent into the venous system. ${ }^{1,2,4,9,11,22,35,38}$ A venous stent should be a compliant scaffold. ${ }^{1}$

The ideal venous scaffold should be crush-resistant too. It is subjected to external and internal pressure. These forces can induce malposition or migration of the stent. The risk increases if external pressure is irregular along the stent, such as in nutcracker or May-Thurner syndromes. To avoid the problem, the stent should have a long length too. ${ }^{2,39}$ Finding stents with long lengths represents a problem because long stents are not eligible for the arterial system. Placing a stent longer than the arterial lesion is dangerous, so there are not a lot of long stents available..$^{2,411,22}$ To cope with it, stents are placed with overlaps, to cover the entire length, but it induces rigidity. ${ }^{4}$

In the arterial system, stenting across joints should be avoided because of the risk of fracture, while in the venous system there is not data of stent's fractures in the same segment. The placement of a venous stent across the inguinal ligament should represent a risk factor for early restenosis. In the cervical region, the venous stent's fracture could be induced by the large movements of the head and the strong muscles. ${ }^{16,22,38,40,46,47}$

Another characteristic of the venous stent is flexibility. Ideally, the scaffold should adapt its shape to the vein, not vice versa. The stent is often quite rigid, because flexibility isn't an important feature in the arterial stent. It causes the vein's straightening and it is not safe. ${ }^{2,4}$ The ideal venous stent should be both flexible and with sufficiently high radial force. ${ }^{4}$

The material should be radiopaque, to follow-up through magnetic resonance and computed tomography. It should be corrosion resistant and fatigue resistant too. ${ }^{4}$

The venous stent should be uncovered, in order to avoid the occlusion of collaterals. $^{2}$

[Veins and Lymphatics 2021; 10:9739]

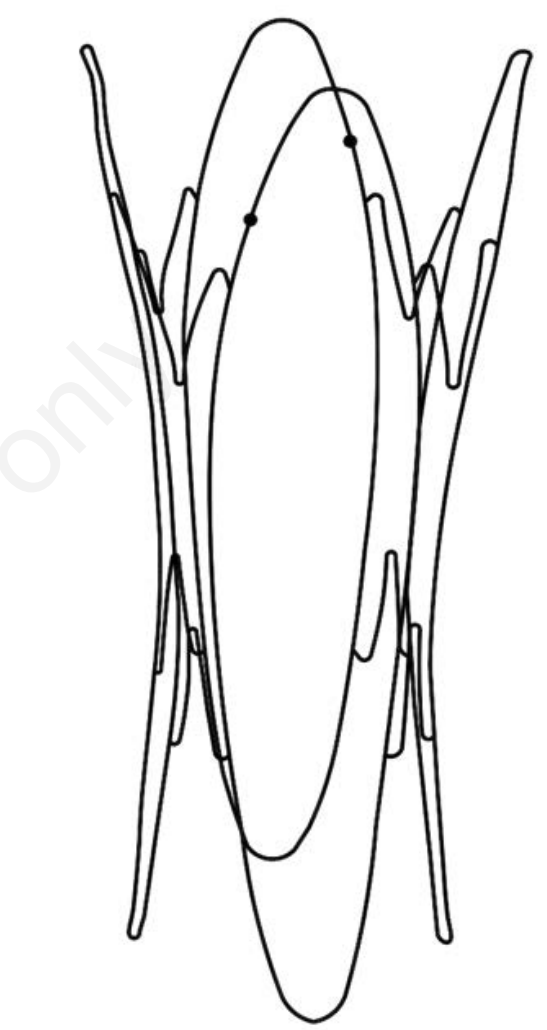

Figure 1. Petalo CVS scaffold. The side close to the heart is larger than the distal one. 40

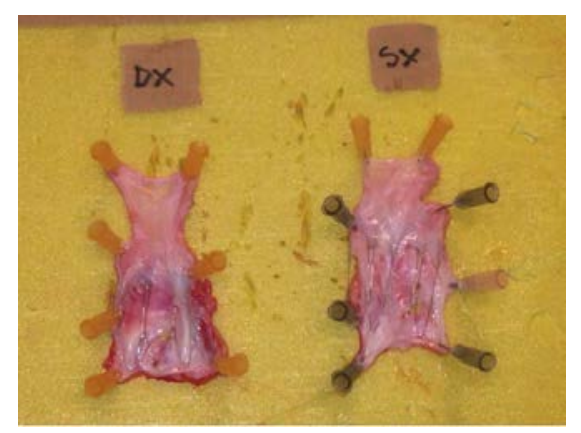

Figure 2. Porcine internal jugular veins implanted with Petalo CVS for six months. The distal metal bars of the device are enveloped in the intimal surface and sometimes the central part is also surrounded. There are not fibrosis and neo-vascularized tissue. ${ }^{40}$ 


\section{Dedicated venous stents}

Venous stents were not available until very recently. For example, Zilver Vena stent, Vici venous stent and Abre venous self-expanding stent are specific for the venous system. ${ }^{4,27,34}$ There is a preclinical study about a new venous scaffold implanted in the porcine internal jugular vein (IJV), that is a compliant nitinol stent, denominated Petalo CVS (Figure 1). ${ }^{40}$

This scaffold has a particular shape, that permits to anchor the vein wall only at the extremities. Its radial force is enough to avoid migration, but it does not cause myointimal hyperplasia. The diameter in the distal part of the stent is smaller than that near the heart, to comply with the veins' anatomy. Thanks to its characteristics, it preserves the venous compliance and the trauma of the vein's wall is extremely low. So the inflammatory response to the placement of the stent is highest after three months and then decreases over time., 90 After three months a neo-intimal tissue covered up the stent's extremities, while the central part was surrounded after six month (Figure 2). ${ }^{9,40}$

\section{New areas of application}

There are in the literature some cases of venous obstruction described recently, that could be treated efficiently with a dedicated venous stent.

One of these is the jugular variant of the Eagle syndrome, caused by the elongation of the styloid process that compresses the IJV. ${ }^{48}$ Another one is JEDI syndrome, where the IJV is subjected to extrinsic and bilateral compression by the omohyoid muscle. ${ }^{49} \mathrm{~A}$ dedicated venous stent could also treat the jugular lesions of the chronic cerebrospinal venous insufficiency (CCSVI) that result unfavorable for balloon angioplasty according to Giaquinta classification. ${ }^{50,51}$

These syndromes are characterized by extrinsic compression, caused by different elements and they could be eligible for venous stenting. ${ }^{48,49,51}$

\section{Conclusions}

Occlusive venous disease can be treated with a stent. ${ }^{3,9-13}$ Venous stents are safe, efficacy and minimally invasive, but historically they are not designed for the venous system. Dedicated venous stents were not available until very recently. 4,9,27,33-36,40 The preclinical study about a new scaffold, denominated Petalo CVS, has been shown that it could have the ideal characteristics of a venous stent, but it is necessary a first in man study to affirm it. ${ }^{9,40}$

\section{References}

1. Zamboni P, Tavoni V, Sisini F, et al. Venous compliance and clinical implications. Veins and Lymphatics 2018;7:7367.

2. Schwein A, Georg Y, Lejay A, et al. Endovascular treatment for venous diseases: where are the venous stents? Methodist Debakey Cardiovasc J. 2018;14:208-13.

3. Raju S, Neglén P. Percutaneous recanalization of total occlusions of the iliac vein. J Vasc Surg 2009;50:360-8.

4. Shamini-Noori SM, Clark TWI. Venous stents: current status and future directions. Tech Vasc Interv Radiol 2018;21:113-16.

5. Li N, Mendoza F, Rugonyi S, et al. Venous biomechanics of angioplasty and stent placement: implications of the poisson effect. J Vasc Interv Radiol 2020;31:1348-56.

6. Eberhardt RT, Raffetto JD. Chronic venous insufficiency. Circulation 2014;130:333-46.

7. Fu J, Tang B, Wang H, et al. Stent characteristics of 32 patients with early $(<14$ days) iliofemoral stent occlusion. J Vasc Surg Venous Lymphat Disord 2020;S2213-333X(20)30556-4

8. Villalba L. Chronic venous insufficiency: the role of venous obstruction and treatment options. Medicine Today 2017;18:33-8.

9. Veroux P, Giaquinta A, Virgilio C, et al. Novel compliant scaffold with specific design for venous system: results of a porcine model study. Biomed Res Int 2018;2018:7312315.

10. Titus JM, Moise MA, Bena J, et al. Iliofemoral stenting for venous occlusive disease. J Vasc Surg 2011;53:70612.

11. Raju S. Treatment of iliac-caval outflow obstruction. Semin Vasc Surg 2015;28:47-53

12. Kölbel T, Lindh M, Akesson M, et al. Chronic iliac vein occlusion: midterm results of endovascular recanalization. $\mathrm{J}$ Endovasc Ther 2009;16:483-91.

13. Qiu P, Zha B, Xu A, et al. Systematic review and meta-analysis of iliofemoral stenting for post-thrombotic syndrome. Eur J Vasc Endovasc Surg 2019;57:40716.

14. Fatima J, AlGaby A, Bena J, et al.
Technical considerations, outcomes, and durability of inferior vena cava stenting. J Vasc Surg Venous Lymphat Disord 2015;3:380-8.

15. Kurklinsky AK, Bjarnason H, Friese JL, et al. Outcomes of venoplasty with stent placement for chronic thrombosis of the iliac and femoral veins: single-center experience. J Vasc Interv Radiol 2012;23:1009-15.

16. Neglén P, Hollis KC, Olivier J, Raju S. Stenting of the venous outflow in chronic venous disease: long-term stent-related outcome, clinical, and hemodynamic result. J Vasc Surg 2007;46:979-90.

17. Garg N, Gloviczki P, Karimi KM, et al. Factors affecting outcome of open and hybrid reconstructions for nonmalignant obstruction of iliofemoral veins and inferior vena cava. J Vasc Surg 2011;53:383-93.

18. Jayaraj A, Raju S. Stenting for obstructive iliac vein lesions. Veins and Lymphatics 2017;6:6855.

19. Petrov I, Grozdinski L, Kaninski G, et al. Safety profile of endovascular treatment for chronic cerebrospinal venous insufficiency in patients with multiple sclerosis. J Endovasc Ther 2011;18:314-23.

20. Mandato KD, Hegener PF, Siskin GP, et al. Safety of endovascular treatment of chronic cerebrospinal venous insufficiency: a report of 240 patients with multiple sclerosis. J Vasc Interv Radiol 2012;23:55-9.

21. Raju S, Hollis K, Neglen P. Obstructive lesions of the inferior vena cava: clinical features and endovenous treatment. J Vasc Surg 2006;44:820-7.

22. Gasparis AP, Labropoulos N. Venous outflow obstruction managing intervention after iliofemoral thrombolysis. Endovascular Today 2011;3:51-6.

23. Razavi MK, Jaff MR, Miller LE. Safety and effectiveness of stent placement for iliofemoral venous outflow obstruction: systematic review and meta-analysis. Circ Cardiovasc Interv 2015;8:e002772.

24. Dabir D, Feisst A, Thomas D, et al. Physical properties of venous stents: an experimental comparison. Cardiovasc Intervent Radiol 2018;41:942-50.

25. Razavi M, Black S, Gagne $P$, et al. Pivotal study of endovenous stent placement for symptomatic iliofemoral venous obstruction. Circ Cardiovasc Interv 2019;12:e08268.

26. George R, Verma H, Ram B, Tripathi R. The effect of deep venous stenting on healing of lower limb venous ulcers. Eur J Vasc Endovasc Surg 2014;48:330- 
6.

27. O'Sullivan GJ, Sheehan J, Lohan D, McCann-Brown JA. Iliofemoral venous stenting extending into the femoral region: initial clinical experience with the purpose-designed Zilver Vena stent. J Cardiovasc Surg 2013;54:255-61.

28. Wen-da W, Yu Z, Yue-xin C. Stenting for chronic obstructive venous disease: A current comprehensive meta-analysis and systematic review. Phlebology 2016;31:376-89.

29. Aurshina A, Ganelin A, Hingorani A, et al. Clinical correlation of the area of inferior vena cava, iliac and femoral veins for stent use. Vascular 2018;26:126-31.

30. Jayaraj A, Crim W, Knight A, Raju S. Characteristics and outcomes of stent occlusion after iliocaval stenting. J Vasc Surg Venous Lymphat Disord 2019;5664.

31. Daugherty SF and Gillespie DL. Venous angioplasty and stenting improve pelvic congestion syndrome caused by venous out ow obstruction. J Vasc Surg Venous Lymphat Disord 2015;3:283-9.

32. Scerrati A, Menegatti E, Zamboni M, et al. Internal jugular vein thrombosis: etiology, symptomatology, diagnosis and current treatment. Diagnostics (Basel) 2021;11:378.

33. Andersen PE, Midtgaard A, Brenøe AS, et al. A new nitinol stent for use in superior vena cava syndrome. Initial clinical experience. J Cardiovasc Surg 2015;56:877-8.

34. Razavi M, Marston W, Black S, et al. The initial report on 1-year outcomes of the feasibility study of the VENITI VICI VENOUS STENT in symptomatic iliofemoral venous obstruction. J Vasc Surg Venous Lymphat Disord
2018;6:192-200.

35. De Wolf MA, de Graaf R, Kurstjens $\mathrm{RL}$, et al. Short-term clinical experience with a dedicated venous nitinol stent: initial results with the sinus-venous stent. Eur J Vasc Endovasc Surg 2015;50:518-26.

36. Stuck AK, Kunz S, Baumgartner I, Kucher N. Patency and clinical outcomes of a dedicated, self-expanding, hybrid oblique stent used in the treatment of common iliac vein compression. J Endovasc Ther 2017;24:159-66.

37. Raju S, Davis M. Anomalous features of iliac vein stenosis that affect diagnosis and treatment. J Vasc Surg Venous Lymphat Disord 2014;2:260-7.

38. Neglén P, Tackett TP Jr, Raju S. Venous stenting across the inguinal ligament. J Vasc Surg 2008;48:1255-61.

39. Hartung O, Grisoli D, Boufi M, et al. Endovascular stenting in the treatment of pelvic vein congestion caused by nutcracker syndrome: lessons learned from the first five cases. J Vasc Surg 2005;42:275-80.

40. Zamboni P, Giaquinta A, Rimondi E, et al. A novel endovenous scaffold for the treatment of chronic venous obstruction in a porcine model: histological and ultrastructural assessment. Phlebology 2019;34:336-46.

41. Jayaraj ACW, Murphy EH, Raju S. Occlusion following iliocaval stenting characteristics and outcomes. J Vasc Surg 2016;63:53S-54S.

42. Grøtta O, Enden T, Sandbæk G, et al. Infrainguinal inflow assessment and endovenous stent placement in iliofemoral post-thrombotic obstructions. CVIR Endovasc 2018;1:29.

43. Zamboni P, Galeotti R, Menegatti E, et al. Chronic cerebrospinal venous insufficiency in patients with multiple scle- rosis. J Neurol Neurosurg Psychiatry 2009;80:392-99.

44. Sisini F, Tessari M, Gadda G, et al. An ultrasonographic technique to assess the jugular venous pulse: a proof of concept. Ultrasound Med Biol 2015;41:1334-41.

45. Zamboni P, Malagoni AM, Menegatti E, et al. Central venous pressure estimation from ultrasound assessment of the jugular venous pulse. PLoS One 2020; 15:e0240057.

46. Seager MJ, Busuttil A, Dharmarajah B, Davies AH. A systematic review of endovenous stenting in chronic venous disease secondary to iliac vein obstruction. Eur J Vasc Endovase Surg 2016;51:100-20.

47. Jayaraj A, Raju S. The venous stent: is it primetime yet? Curr Manage Venous Dis 2017;489-96.

48. Zamboni P, Scerrati A, Menegatti E, et al. The eagle jugular syndrome. BMC Neurol 2019;19:333.

49. De Bonis P, Menegatti E, Cavallo MA, et al. JEDI (jugular entrapment, dilated ventricles, intracranial hypertension) syndrome: a new clinical entity? A case report. Acta Neurochir (Wien) 2019;161:1367-70.

50. Giaquinta A, Beggs CB, Veroux M, et al. Factors influencing the hemodynamic response to balloon angioplasty in the treatment of outflow anomalies of internal jugular veins. J Vasc Surg Venous Lymphat Disord 2017;5:777-88.

51. Zamboni P, Galeotti R, Salvi F, et al. Effects of venous angioplasty on cerebral lesions in multiple sclerosis: expanded analysis of the brave dreams double-blind, sham-controlled randomized trial. J Endovasc Ther 2020;27:1526602819890110. 